Rev. Int. Contam. Ambie. 35 (4) 787-796, 2019

DOI: 10.20937/RICA.2019.35.04.01

\title{
EVALUACIÓN DE RIESGOS A LA SALUD DE LACTANTES EXPUESTOS A METALES TRAZA EN LECHE MATERNA
}

Health risk assessment of children by maternal milk consumption with content of heavy metals

\author{
Andrés Mauricio ZAPATA-RIVERA ${ }^{1 *}$, Pauly Melissa QUIMBAYO ${ }^{2}$, \\ Fabian MENDÉZZ ${ }^{3}$, Jenny Elizabeth ORDOÑEZ-BETANCOURTH ${ }^{3}$, \\ Nathalie ABRAHAMS-CHOW ${ }^{3}$ y Martha Isabel PÁEZ-MELO ${ }^{2}$
}

${ }^{1}$ Grupo de investigación en Saneamiento Ambiental, Instituto Cinara, Universidad del Valle, sede Melendéz, calle 13 núm. 100-00, edificio E37, Cali, Colombia.

${ }^{2}$ Grupo de Investigación en Contaminación Ambiental por Metales y Plaguicidas, Universidad del Valle, sede Melendéz, calle 13 núm. 100-00, edificio 320, Cali, Colombia

${ }^{3}$ Grupo Epidemiología y Salud Poblacional, Universidad del Valle, sede San Fernando, calle 4B núm. 36-00, edificio 118, Cali, Colombia

*Autor para correspondencia: andres.zapata@correounivalle.edu.co

(Recibido: diciembre 2017; aceptado: diciembre 2018)

Palabras clave: río Cauca, cadmio, mercurio, plomo, salud ambiental, estudios poblacionales

\section{RESUMEN}

El objetivo de este estudio fue evaluar el riesgo en la salud de lactantes de menos de un mes a 24 meses de edad por ingesta de leche materna con contenido de cadmio (Cd), mercurio $(\mathrm{Hg})$ y plomo $(\mathrm{Pb})$. Se cuantificó el riesgo de efectos no carcinogénicos (REN) usando modelación probabilística con el programa Crystal Ball ${ }^{\circledR}$, siguiendo los lineamientos del modelo de la Agencia de Protección Ambiental de Estados Unidos (USEPA). El promedio de los niveles máximos medidos de $\mathrm{Cd}(8.87 \mu \mathrm{g} / \mathrm{kg}), \mathrm{Hg}(20.88 \mu \mathrm{g} / \mathrm{kg})$ y Pb $(37.29 \mu \mathrm{g} / \mathrm{kg})$ estuvo 5, 21 y 19 veces, respectivamente, por encima de los límites máximos permitidos por la Organización Mundial de la Salud. Con relación al riesgo, los valores asociados con el contenido de al menos uno de los metales en leche materna pudieran superar en cada lugar de muestreo y cada subgrupo de lactantes los límites máximos permitidos por la USEPA (REN $\geq 1)$. Se presentó la primera evidencia de contaminación por metales pesados en leche materna de mujeres que viven en la ribera del río Cauca. En casos aislados, los lactantes expuestos por ingesta de esta leche, exceden el consumo tolerable de dichos contaminantes. Tomando en cuenta el peor escenario, los niños de la zona presentan riesgo no cancerígeno superior al máximo permitido. Se confirma la necesidad de acciones de mitigación para reducir potenciales riesgos a la salud de los lactantes. Esto no debe conducir a la sustitución de la lactancia como medida adecuada, sino a establecer políticas integrales que disminuyan las inequidades ambientales y de salud.

Key words: Cauca river, cadmium, mercury, lead, environmental health, population studies 


\begin{abstract}
The aim of this study was to assess the health risk of infants between 0 and 24 months of age due to ingestion of breast milk containing cadmium $(\mathrm{Cd})$, mercury $(\mathrm{Hg})$ and lead $(\mathrm{Pb})$. The non-carcinogenic risk (NCR) was quantified using probabilistic models with the Crystal Ball ${ }^{\circledR}$ program, following the model guidelines of the United States Environmental Protection Agency (USEPA). The average of the maximum measured levels of $\mathrm{Cd}$ ( 8.87 $\mu \mathrm{g} / \mathrm{kg}) \mathrm{Hg},(20.88 \mu \mathrm{g} / \mathrm{kg})$ and $\mathrm{Pb}(37.29 \mu \mathrm{g} / \mathrm{kg})$ was 5, 21 and 19 times, respectively, above the maximum limits allowed by the World Health Organization. Regarding risk, the values associated with the content of at least one of the metals in breast milk could exceed the maximum limits allowed by the USEPA at each sampling site and each subgroup of babies $(\mathrm{NCR} \geq 1)$. The first evidence of contamination by heavy metals in the breast milk of women living on the banks of the Cauca river was presented. In isolated cases, babies exposed by the intake of this milk, exceed the tolerable consumption of these contaminants. Taking into account the worst scenario, children in the area present a non-carcinogenic risk higher than the maximum allowed. The need of mitigation actions is confirmed for reducing potential risks to the health of infants. This should not lead to replace breastfeeding as an adequate measure, but to establish comprehensive policies aimed to reduce environmental and health inequities.
\end{abstract}

\section{INTRODUCCIÓN}

De acuerdo con la Organización Mundial de la Salud (OMS), la lactancia materna es la forma ideal de alimentación para los niños pequeños (Solomon y Weiss 2002). La lactancia es esencial para que los niños alcancen todo su potencial en relación con su crecimiento, salud y desarrollo. La OMS estima que el logro de la cobertura universal de una lactancia materna óptima, podría evitar globalmente el $13 \%$ de las muertes que ocurren en los niños menores de 5 años (OMS 2010). Sin embargo, el contenido de grasas y proteínas de la leche materna puede hacer de esta una ruta de excreción de agentes tóxicos, ya que la leche materna se convierte en ruta de exposición de agentes contaminantes como los metales pesados (LaKind et al. 2004). Por ello los lactantes son una de las poblaciones más vulnerables frente a los efectos de la contaminación ambiental

Las comunidades que se asientan sobre la ribera de los ríos o zonas costeras tienden a basar su dieta proteínica en un mayor consumo de pescado. Se ha demostrado que estas comunidades pueden consumir entre 40 y $50 \%$ más pescado que el resto de la población (Castro y Méndez 2008). El pescado es una reconocida ruta de exposición a metales pesados como cadmio, mercurio y plomo (Vieira et al. 2011, Álvarez et al. 2012). Estudios realizados en España, específicamente en poblaciones costeras o asentadas sobre la ribera de los ríos, establecieron que hábitos alimenticios como el consumo de pescado están asociados con la presencia de metales pesados en leche materna (García et al. 2011); debido a ello, las mujeres lactantes expuestas a estos contaminantes los absorben vía el tracto gastrointestinal y los transfieren de la sangre a la leche materna (Grandjean et al. 1994). A pesar de estas desventajas, en el informe de la consulta mixta de expertos de la Organización de las Naciones Unidas para la Alimentación y la agricultura (FAO, por sus siglas en inglés) y la OMS sobre los riesgos y los beneficios del consumo de pescado (FAO-OMS 2010) se concluyó que ante eventos de contaminación ambiental en pescado, restringir por completo el consumo no debería ser una acción de carácter permanente para disminuir el riesgo en la salud de una población. Esto, debido a beneficios como el aporte energético, proteínico y de nutrientes como los ácidos grasos poliinsaturados de cadena larga n-3 (AGPICLn3), que son característicos de este tipo de proteína animal; asimismo, a que en mujeres en edad fértil, el consumo de pescado reduce el riesgo de deficiencias del desarrollo neurológico en la progenie, siempre que la exposición materna a contaminantes ambientales (como dioxinas o metales pesados) por esta vía no supere el valor de la ingesta mensual provisional tolerable. Se ha demostrado que la etapa fetal y la de lactancia son los periodos más susceptibles a los efectos en salud por la exposición a metales pesados como el mercurio $(\mathrm{Hg})$, el plomo $(\mathrm{Pb})$ y el cadmio $(\mathrm{Cd})$, ya que en este periodo el porcentaje de absorción de estos compuestos es mayor al que se presenta en la etapa adulta (Mata et al. 2003). En la población infantil, la contaminación por estos metales se ha asociado con alteraciones en el 
desarrollo normal del cerebro y el sistema nervioso central, reducciones del cociente de inteligencia (IQ) y malformaciones congénitas (Dietrich et al. 1993, Thompson y Bannigan 2008).

Esta problemática se acentúa debido a que los ríos son receptores de gran parte de la contaminación generada en los grandes centros urbanos. En Colombia, el río Cauca y sus afluentes se caracterizan por ser receptores de las descargas de aguas residuales de las poblaciones ubicadas a lo largo de su recorrido. Estas poblaciones incluyen los mayores centros urbanos del suroccidente colombiano: Popayán, Cali y Yumbo. Además, debido al crecimiento industrial, la minería y el mal manejo de las basuras, este río es receptor de desechos industriales, aguas residuales y lixiviados en los que se han identificado metales pesados y pesticidas, los cales ocasionan graves efectos sobre la biota acuática (Vivas et al. 2014).

A su paso por la ciudad de Cali, el río Cauca recoge parte de la contaminación generada en el antiguo vertedero de residuos sólidos de Navarro (AVN), que fue clausurado 2008 pero aún continua vertiendo lixiviados. El AVN es un generador de contaminación reconocido para la ciudad de Cali y el río Cauca. Se han documentado emisiones de benceno con concentraciones por encima de la norma colombiana, y elevadas concentraciones de metano y material particulado menor a $5 \mu \mathrm{m}$. Se ha documentado que éstos pueden generar efectos ambientales negativos en la salud humana en áreas pobladas a menos de $3 \mathrm{~km}$ (Gómez et al. 2008)el Botadero de Navarro (BN. La exposición al vertedero se ha asociado con disminución en la función pulmonar y mayor prevalencia y cronicidad de síntomas respiratorios en personas mayores de 50 años (Filigrana et al. 2011). En niños entre 1 y 5 años de edad, se ha encontrado mayor riesgo de presentar síntomas respiratorios y bajo peso al nacer (de Burbure et al. 2006). También se ha analizado el potencial teratogénico de los lixiviados del AVN y su efecto sobre las especies acuáticas del rio Cauca (Castilla et al. 2008, Orioli et al. 2009, Nazer et al. 2011). Estudios realizados en peces provenientes del río Cauca, documentaron una alta bioacumualción de los metales pesados cadmio, mercurio y plomo en el músculo de dos especies de peces, Prochilodus magdalenae (bocachico) y Chaetostoma spp. (corroncho) (Artunduaga 2014), a su paso por el corregimiento el Hormiguero y la Comuna 6 al suroriente de Cali, así como la vereda El Paso de la Torre en el municipio de Yumbo.

Por las razones expuestas, el objetivo de este estudio fue evaluar el riesgo en la salud de lactantes de 0 a 24 meses de edad expuestos a cadmio, mer- curio y plomo por consumo de leche materna en tres poblaciones ubicadas sobre la ribera del río Cauca. Éstas son: el corregimiento El Hormiguero y el Jarillón de la Comuna 6 en la ciudad de Cali, y la vereda el Paso de la Torre en el municipio de Yumbo. La evaluación del riesgo en salud es una aproximación desde las ciencias naturales a la identificación de los agentes ambientales que representan un peligro, la estimación de la exposición a dichos agentes y la caracterización de los riesgos a la salud humana. En particular, esta investigación se basó en el modelo conceptual del riesgo total, un modelo lineal que empieza en la fuente de contaminación, la cual emite los contaminantes al suelo, aire o agua, y a partir de allí configura la potencial exposición de los seres vivos a través de rutas de exposición como la ingestión, el contacto dérmico o la inhalación (Páez et al. 2011).

\section{MATERIALES Y MÉTODOS}

Se realizó un estudio de evaluación de riesgo en la salud humana de acuerdo con el Programa Internacional de Seguridad Química (IPCS, por sus siglas en inglés). La evaluación incluyó cuatro etapas: identificación y caracterización del peligro, medición de la exposición y caracterización del riesgo. El estudio se enfocó a las dos últimas etapas, a partir de las cuales se determinó la concentración del contaminante en muestras de leche materna y se estimó el riesgo no cancerígeno en el grupo receptor. Este riesgo es una función de la toxicidad de la sustancia peligrosa evaluada y la magnitud y el tiempo de exposición a la misma (USEPA 2001), lo cual representa una propuesta complementaria a los análisis de riesgo en salud basados en estudios epidemiológicos. De acuerdo con la recomendación de la OMS para compuestos químicos peligrosos, este tipo de estudios constituyen otro soporte para la toma de decisiones en salud pública. Para el estudio se contó con el aval del comité de ética de la Universidad del Valle y el consentimiento informado de las madres que participaron.

\section{Ubicación y caracterización de la zona de estudio}

Las poblaciones fueron seleccionadas por su cercanía con el mayor centro poblado del suroccidente colombiano (la ciudad de Cali), el antiguo vertedero de residuos sólidos a cielo abierto de Navarro y el mayor centro industrial de esta zona del país (el municipio de Yumbo). De acuerdo con las personerías (entidades de servicio público que vigilan y hacen control sobre la gestión de las alcaldías y 
entes descentralizados) del municipio de Cali y del departamento del Cauca, esta zona ha evidenciado un incremento de la minería ilegal, particularmente en la zona del Parque Nacional Natural los Farallones de Cali y el norte del departamento del Cauca, con la consecuente contaminación con mercurio de fuentes hídricas como los ríos Cali y Meléndez en la ciudad de Cali y de los ríos Ovejas, Teta y Quinamayó en el norte del departamento del Cauca, todos tributarios del río Cauca. En ese sentido, se configuraron tres zonas de estudio: 1) Corregimiento el Hormiguero, el cual se encuentra localizado al sur de la ciudad de Cali, a $16 \mathrm{~km}$ de la zona urbana de la ciudad y a 5 km de Navarro; 2) Jarillón del río Cauca, Comuna 6 , un dique que se encuentra ubicado en la ciudad de Cali sobre la margen izquierda del río Cauca a
$10 \mathrm{~km}$ del vertedero de Navarro; 3 ) vereda Paso de la Torre, localizada hacia el norte, a $4.5 \mathrm{~km}$ de la ciudad de Yumbo. Las tres zonas se caracterizan por tener población con bajos ingresos económicos, un nivel educativo entre primaria y secundaria y por su consumo permanente de pescado proveniente del río Cauca (Echeverry et al. 2015, Figueroa et al. 2017). La figura 1 muestras la zona de estudio.

\section{Muestra poblacional}

En la zona se encontraron 22 madres lactantes con bebés de 0 a 24 meses de edad (El Hormiguero, $\mathrm{N}=5$; Comuna $6, \mathrm{~N}=13$; vereda Paso de la Torre $\mathrm{N}=4$ ), quienes al momento del estudio consumían leche materna. Las 22 madres lactantes y sus bebés correspondieron al total de madres lactantes residentes en

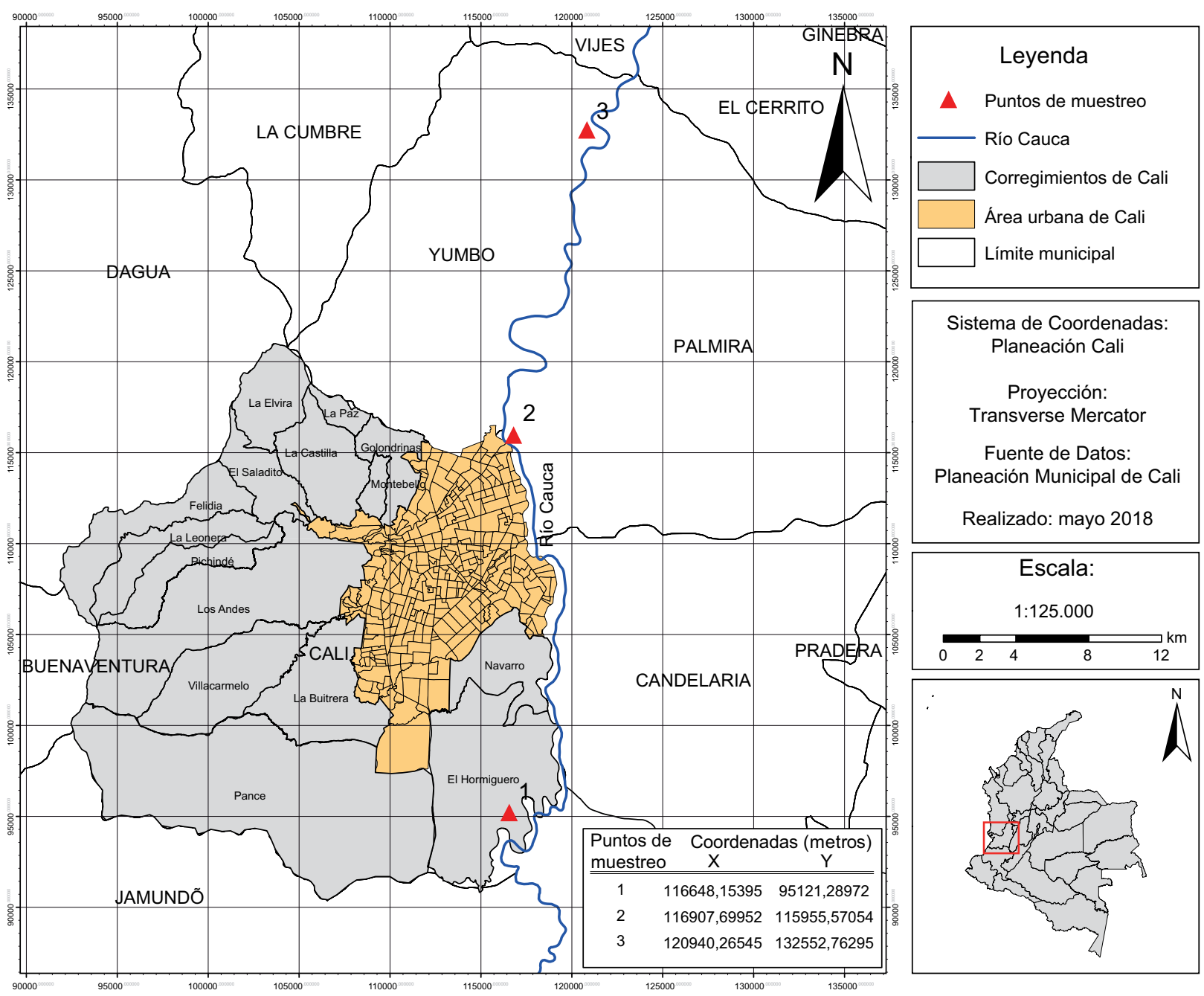

Fig. 1. Ubicación de la zona de estudio. (1) Corregimiento El Hormiguero, (2) Jarillón Floralia, Comuna 6, (3) vereda Paso de la Torre 
la zona al momento de este estudio. La muestra fue una muestra propositiva de madres y niños lactantes que asistían regularmente a los controles médicos en el centro de salud colaborador. La asistencia regular de las madres a los controles garantizaba su presencia durante los procesos del estudio, tales como toma del consentimiento informado, educación relacionada con la toma de muestras biológicas y selección del mejor día para hacer la toma de leche materna.

\section{Muestreo de leche materna}

La toma de muestras de leche materna se realizó en un muestreo puntual durante una temporada atípica de alta lluvia, entre los meses de diciembre a febrero de 2014 y 2015, respectivamente. La recolección de las muestras se realizó con un extractor manual de leche materna con personal especializado de acuerdo con el procedimiento operativo estándar para el muestreo de compuestos orgánicos persistentes (COP) en leche materna (Heidelore et al. 2010). El volumen colectado por muestra fue $60 \mathrm{~mL}$. Las muestras fueron depositadas en recipientes plásticos con tapa hermética, previamente lavados y secados en el laboratorio. Posteriormente, los frascos fueron puestos en bolsas con sello hermético debidamente rotuladas. Se transportaron en neveras con geles refrigerantes y se realizó un blanco de transporte para evaluar la calidad de las mediciones y evitar contaminación cruzada. Entre la toma de la muestra y su almacenamiento en el laboratorio transcurrió un tiempo de $4 \mathrm{~h}$.

\section{Pretratamiento y preservación de las muestras}

La temperatura de transporte de las muestras se mantuvo controlada a $4{ }^{\circ} \mathrm{C}$ hasta su llegada al laboratorio. Posteriormente, las muestras se congelaron y liofilizaron a $-40{ }^{\circ} \mathrm{C}$ durante $32 \mathrm{~h}$. La liofilización se llevó a cabo en el laboratorio de la Escuela de Ingeniería de Alimentos de la Universidad del Valle utilizando un liofilizador marca Labconco (LabconcoCorporation, Kansas City, EUA).

\section{Cuantificación de los metales pesados en leche materna}

Cada muestra de leche materna se analizó por triplicado. El método de extracción para los analitos fue la digestión ácida asistida por microondas, según las especificaciones del método de la Agencia de Protección Ambiental de los Estados Unidos (USEPA, por sus sigles en inglés): USEPA 3052 (Tobergte y Curtis 2013). La cuantificación de los metales se realizó siguiendo el método USEPA 7010 (USEPA 1998), utilizando espectrometría de absorción atómica. Los metales cadmio y plomo se analizaron por absorción atómica en horno de grafito, mientras que el mercurio se cuantificó por vapor frío. Se utilizó un equipo Shimadzu AA 6300 (Shimadzu Inc., Riverwood Drive, Columbia, USA). Todos los reactivos utilizados fueron de grado analítico y los estándares para cadmio, mercurio y plomo y $\mathrm{Pb}$ fueron marca Sigma Aldrich (San Luis, EUA) con una concentración de $1000 \mathrm{mg} / \mathrm{L}$ en ácido nítrico cada uno. Se obtuvieron los siguientes límites de detección: $\mathrm{Cd}=0.01 \mu \mathrm{g} / \mathrm{kg}$, $\mathrm{Hg}=0.16 \mu \mathrm{g} / \mathrm{kg}$ y $\mathrm{Pb}=0.60 \mu \mathrm{g} / \mathrm{kg}$.

\section{Escenario de la evaluación de riesgo a la salud humana}

Para los propósitos de este estudio, la leche materna con contenido de cadmio, mercurio y plomo se consideró la ruta a través de la cual los contaminantes llegan a la población en estudio, siendo la ingestión la vía a través de la cual los contaminantes llegan al sistema gastrointestinal de la población receptora.

Para la estimación del riesgo a la salud de los lactantes se utilizaron los modelos de la USEPA. Según éstos, el riesgo es una función de la toxicidad de la sustancia evaluada, la magnitud y el tiempo de exposición. Esta última es una medida de la calidad y cantidad del contacto entre la sustancia y el organismo expuesto (USEPA 2005).

\section{Ruta de exposición por ingestión}

La ingesta (I), se determinó utilizando la ecuación genérica para el cálculo de la exposición, expresada a menudo como un consumo diario promedio basado en el peso corporal (unidades de miligramos por kilogramo de peso corporal por día [mg/kg $\times$ día]). La ecuación genérica fue la siguiente (USEPA 2005):

$I=\frac{C \times I R \times C F \times E F \times E D}{B w \times A T}$

donde $C$ es la concentración en la muestra $(\mathrm{mg} / \mathrm{kg})$, $I R$ la tasa de ingestión de la muestra (mg/día), $C F$ el factor de conversión (1E-06 kg/mg), la f, I la duración de la exposición (años), IW el peso corporal $(\mathrm{kg})$ y $A T$ el tiempo promedio (días).

\section{Obtención de los parámetros del modelo para la evaluación del riesgo en salud}

La encuesta para obtener información nutricional de las madres fue adaptada culturalmente a la población de este estudio. Para la evaluación de riesgo, los niños se dividieron en subgrupos debido a los cambios drásticos que experimentan los bebés en variables como el peso corporal y el consumo de leche materna. En el cuadro I se presentan el 
CUADRO I. PARÁMETROS DEL MODELO DE LA AGENCIA DE PROTECCIÓN AMBIENTAL DE ESTADOS UNIDOS

\begin{tabular}{|c|c|c|c|c|c|c|c|}
\hline \multirow{2}{*}{ Subgrupo } & \multicolumn{2}{|c|}{$\begin{array}{l}\text { Consumo de leche } \\
\text { materna (mg/día) }\end{array}$} & \multicolumn{2}{|c|}{$\begin{array}{c}\text { Frecuencia de } \\
\text { exposición (días/año) }\end{array}$} & \multirow{2}{*}{$\begin{array}{c}\text { Duración de la } \\
\text { exposición (años) }\end{array}$} & \multicolumn{2}{|c|}{$\begin{array}{c}\text { Peso } \\
\text { corporal }(\mathrm{kg})\end{array}$} \\
\hline & Mínimo & Máximo & Mínimo & Máximo & & Mínimo & Máximo \\
\hline $0-3$ & 7.95E05 & $9.17 \mathrm{E} 05$ & 1.00 & 7.50 & 0.25 & 3.40 & 6.40 \\
\hline $3-6$ & $9.17 \mathrm{E} 05$ & $9.48 \mathrm{E} 05$ & 3.75 & 20.0 & 0.50 & 6.20 & 8.64 \\
\hline $6-12$ & $8.56 \mathrm{E} 05$ & $1.28 \mathrm{E} 06$ & 6.34 & 45.6 & 1.00 & 6.40 & 11.7 \\
\hline $12-24$ & 7.95E05 & $9.17 \mathrm{E} 05$ & 30.4 & 45.6 & 2.00 & 8.36 & 14.0 \\
\hline
\end{tabular}

intervalo de edad en meses de cada subgrupo y los valores de los parámetros: consumo de leche materna, frecuencia de exposición, duración de la exposición y peso corporal. Todas las unidades son las requeridas en el modelo USEPA.

\section{Caracterización y cálculo probabilístico del riesgo}

Según la USEPA, el cálculo probabilístico del riesgo implica un muestreo aleatorio a partir del cual se generan las distribuciones de las variables incluidas en la ecuación genérica de la ingesta. El análisis Monte Carlo es uno de los métodos probabilísticos más utilizados con este fin, por lo que; por tal razón, para el cálculo del riesgo se siguieron los lineamientos de un modelo probabilístico usando el programa computacional Crystal Ball 11.0® (Decisioneering Inc., Denver, Colorado, EUA.) con simulaciones tipo Monte Carlo para cien mil iteraciones, basadas en los tipos de distribución de probabilidad de cada variable. Para ello se aplicó la prueba de normalidad Shapiro Wilks $(\mathrm{N}<50 \mathrm{y}$ $\mathrm{P}>0.05)$ empleando el programa computacional Statistica v. 8.0 (StatSoft, Poland). Posteriormente se aplicó la prueba de chi cuadrado con el fin de determinar la distribución a la que se ajustaban los datos. La prueba de Shapiro Wilks mostró que no había normalidad en ninguna de las variables de la ecuación, mientras que la prueba de chi cuadrado estableció que todas las variables se ajustaban a una distribución rectangular. Por tanto, se trabajó con los valores máximos y mínimos de cada una de las variables del modelo USEPA.

El cálculo del riesgo por sustancia de efectos tóxicos no carcinogénicos se realizó por el cociente del valor de la ingesta calculada con una dosis de referencia específica. Si se considera que existe un riesgo para la salud inaceptable y habrá que tomar medidas correctivas (USEPA 2005). Los valores de la dosis de referencia (RfD) para cadmio, metil mercurio y plomo fueron $1.00 \times 10^{-3}, 1.00 \times 10^{-4} \mathrm{y}$ $3.57 \times 10^{-3} \mathrm{mg} / \mathrm{kg} \times$ día, respectivamente.

\section{RESULTADOS}

\section{Encuesta poblacional}

Las muestras de leche materna en las que se midió el contenido de cadmio, mercurio y plomo fueron tomadas de madres lactantes entre $\operatorname{los} 15 \mathrm{y}$ 29 años de edad. La encuesta poblacional indicó que el $90 \%$ de ellas vive en estrato socioeconómico 1 , mientras que el $10 \%$ en el estrato socio-económico 2 , es decir, que son madres de escasos recursos económicos con accesos deficientes a servicios de salud y educación.

De acuerdo con la encuesta, $50 \%$ de las madres se identificó como de raza negra, $10 \%$ como indígena, $30 \%$ como mestiza y $10 \%$ como de raza blanca. Del total de madres, $30 \%$ contaba con nivel educativo de primaria básica, $60 \%$ de secundaria de básica y el $10 \%$ restante no contaba con estudios. E1 $90 \%$ de las madres lactantes no fumaba, lo que permitió descartar el cigarrillo como fuente de contaminación por cadmio. El $100 \%$ manifestó que no estaba expuesto a los metales evaluados por causas ocupacionales. La encuesta reveló que el pescado extraído del río Cauca era parte regular de la dieta alimenticia de las mujeres en la zona de estudio. Se logró establecer que la tasa de ingestión de pescado alcanzaba los $7.4 \mathrm{~kg} / \mathrm{año}$, valor un $40 \%$ superior al consumo aparente per capita de $4.5 \mathrm{~kg} / \mathrm{año}$ en Colombia (Restrepo et al. 2016).

\section{Metales en leche materna}

Los valores medidos de plomo, cadmio y mercurio en leche materna se registraron en el cuadro II, donde se consigna el promedio de las tres mediciones hechas a cada muestra.

\section{Evaluación probabilística del riesgo en la salud}

Los resultados probabilísticos para la evaluación del riesgo por efectos no cancerígenos debidos a ingesta de leche materna con contenido de cadmio, mercurio y plomo se registran en el cuadro III. 
CUADRO II. NIVELES DE METALES PESADOS MEDIDOS EN LECHE MATERNA

\begin{tabular}{|c|c|c|c|c|}
\hline Referencia & $\begin{array}{l}\text { Núm. de } \\
\text { Muestra }\end{array}$ & $\begin{array}{l}\text { Plomo } \\
\mu \mathrm{g} / \mathrm{kg}\end{array}$ & $\begin{array}{c}\text { Cadmio } \\
\mu \mathrm{g} / \mathrm{kg}\end{array}$ & $\begin{array}{c}\text { Mercurio } \\
\mu \mathrm{g} / \mathrm{kg}\end{array}$ \\
\hline Hormiguero & $\begin{array}{l}1 \\
2 \\
3 \\
4 \\
5\end{array}$ & $\begin{aligned} & 23.61^{\mathrm{a}} \\
&<0.60 \\
&<0.60 \\
&<0.60 \\
&<0.60\end{aligned}$ & $\begin{array}{c}6.70^{\mathrm{a}} \\
<0.01 \\
5.86^{\mathrm{a}} \\
26.16^{\mathrm{a}} \\
<0.01\end{array}$ & $\begin{array}{c}22.22^{\mathrm{a}} \\
16.50^{\mathrm{a}} \\
28.34^{\mathrm{a}} \\
<0.16 \\
<0.16\end{array}$ \\
\hline Floralia & $\begin{array}{r}6 \\
7 \\
8 \\
9 \\
10 \\
11 \\
12 \\
13 \\
14 \\
15 \\
16 \\
17 \\
18\end{array}$ & $\begin{array}{c}<0.60 \\
<0.60 \\
<0.60 \\
<0.60 \\
<0.60 \\
<0.60 \\
<0.60 \\
<0.60 \\
<0.60 \\
<0.60 \\
50.96^{\mathrm{a}} \\
<0.60 \\
<0.60\end{array}$ & $\begin{aligned} &<0.01 \\
&<0.01 \\
&<0.01 \\
&<0.01 \\
&<0.01 \\
&<0.01 \\
&<0.01 \\
&<0.01 \\
& \quad 4.22^{\mathrm{a}} \\
&<0.01 \\
&<0.01 \\
&<0.01 \\
& \quad 1.40\end{aligned}$ & $\begin{array}{l}39.33^{\mathrm{a}} \\
10.88^{\mathrm{a}} \\
11.09^{\mathrm{a}} \\
11.01^{\mathrm{a}} \\
<0.16 \\
<0.16 \\
<0.16 \\
<0.16 \\
<0.16 \\
<0.16 \\
<0.16 \\
<0.16 \\
<0.16\end{array}$ \\
\hline $\begin{array}{l}\text { Paso de } \\
\text { la Torre }\end{array}$ & $\begin{array}{l}19 \\
20 \\
21 \\
22\end{array}$ & $\begin{array}{l}<0.60 \\
<0.60 \\
<0.60 \\
<0.60\end{array}$ & $\begin{array}{l}<0.01 \\
<0.01 \\
<0.01 \\
<0.01\end{array}$ & $\begin{array}{r}54.77^{\mathrm{a}} \\
6.42^{\mathrm{a}} \\
8.22^{\mathrm{a}} \\
<0.16\end{array}$ \\
\hline $\begin{array}{l}\text { Límites Codex } \\
\text { Alimentarius }\end{array}$ & & 2.00 & 5.00 & 1.00 \\
\hline Límites OMS & & 2.00 & 3.00 & 1.00 \\
\hline
\end{tabular}

${ }^{\mathrm{a}}$ Valor por encima de los límites de la OMS y el Códex Alimentarius de la Unión Europea. Límites de detección: Cd: $0.01 \mu \mathrm{g} / \mathrm{kg}$, Hg: $0.16 \mu \mathrm{g} / \mathrm{kg}, \mathrm{y} \mathrm{Pb}: 0.60 \mu \mathrm{g} / \mathrm{kg}$

\section{DISCUSIÓN}

\section{Encuesta poblacional}

Estudios recientes efectuados en un sector del oriente de la ciudad de Cali, lograron establecer que ciertas condiciones de tipo social, económico y cultural tales como bajo nivel de ingresos económicos, bajo nivel educativo y pertenencia a un determinado tipo de etnia, (afrodescendiente), actúan como determinantes socioeconómicos que favorecen la exposición y posterior contaminación por metales como mercurio, plomo y cadmio, especialmente por el consumo de pescado (en el caso particular de este estudio, extraído del río Cauca). Esto se debe a que es un tipo de proteína de fácil acceso y muy bajo costo (Figueroa et al. 2017). En otras palabras, la encuesta poblacional permitió identificar cierto tipo de condicionantes socioeconómicos en la zona de estudio, que de acuerdo con la Organización Panamericana de la Salud (OPS) limitan el acceso de la población a alimentos de mejor calidad, favorecen el deterioro de los buenos hábitos de vida, limitan el acceso a los servicios de saneamiento básico y generan situaciones de injusticia ambiental que en menor o mayor medida favorecen la exposición de la población a contaminantes ambientales, como los metales evaluados en este trabajo (OPS 2010).

\section{Metales en la leche materna}

Se encontró que el $64 \%$ de las muestras contenía alguno de los tres metales analizados. De este porcentaje, el $14 \%$ se presentó en la vereda el Paso de la Torre, el $32 \%$ en el jarillón de Floralia en la Comuna 6 de Cali y el $18 \%$ en el corregimiento El Hormiguero. Los dos puntos más cercanos al vertedero de Navarro son los que presentan mayor cantidad de muestras con presencia de metales pesados. Esto podría indicar una relación entre ambos factores. Asimismo, la encuesta reveló que las madres corresponden a un grupo de personas no fumadoras y que no están expuestas ocupacionalmente a los metales del estudio; también que entre las madres hay un alto consumo de pescado del río Cauca, por lo cual existe una alta probabilidad de que el contenido de metales en la leche materna se correlacione con el alto consumo de pescado de este río.

En el caso específico del mercurio, distintos autores han destacado los graves problemas de contaminación en los ecosistemas acuáticos colombianos ocasionados por el uso indiscriminado de este metal en la minería legal e ilegal. Lo anterior ha afectado principalmente a organismos acuáticos como los peces (Mancera y Álvarez 2006). Autores como Gracia et al. (2010) lograron correlacionar altas concentraciones de mercurio en cabello de los habitantes del municipio de Ayapel, Córdoba, Colombia, con altos consumos de pescado contaminado con mercurio. Asimismo, Olivero (2015) argumentó que factores como altos niveles de pobreza, abandono estatal, tecnologías obsoletas y falta de reglamentación estatal, aumentan la vulnerabilidad de las poblaciones frente a la contaminación por mercurio. Los resultados de la encuesta revelaron que todos estos factores convergen en las poblaciones estudiadas, razón por la cual cualquier intervención de tipo estatal para disminuir la presencia de contaminantes en la leche materna de las mujeres de la zona debe incluir acciones integrales que favorezcan la disminución de los índices de pobreza, el uso indiscriminado de mercurio y la regulación de la actividad minera. 
CUADRO III. RESULTADOS PARA EL RIESGO NO CANCERÍGENO EN LACTANTES

\begin{tabular}{|c|c|c|c|c|c|c|}
\hline \multirow{2}{*}{$\frac{\text { Referencia }}{\text { Contaminante }}$} & \multicolumn{2}{|c|}{ Hormiguero } & \multicolumn{2}{|c|}{ Comuna 6} & \multicolumn{2}{|c|}{ Paso de la Torre } \\
\hline & Mínimo & Máximo & Mínimo & Máximo & Mínimo & Máximo \\
\hline \multicolumn{7}{|c|}{ Lactantes de $0-3$ meses } \\
\hline Cadmio & $2.27 \mathrm{E}-03$ & $1.34 \mathrm{E}-01$ & $5.13 \mathrm{E}-04$ & $2.23 \mathrm{E}-02$ & $5.00 \mathrm{E}-06$ & $5.00 \mathrm{E}-06$ \\
\hline Mercurio & $5.95 \mathrm{E}-02$ & $1.49 \mathrm{E}+00^{*}$ & 4.54E-02 & $2.08 \mathrm{E}+00^{*}$ & $2.81 \mathrm{E}-02$ & $2.84 \mathrm{E}+00^{*}$ \\
\hline Plomo & $2.36 \mathrm{E}-03$ & $3.63 \mathrm{E}-02$ & 5.09E-03 & $7.83 \mathrm{E}-02$ & $1.00 \mathrm{E}-03$ & $1.00 \mathrm{E}-03$ \\
\hline Suma & $6.41 \mathrm{E}-02$ & $1.66 \mathrm{E}+00^{*}$ & $5.10 \mathrm{E}-02$ & $2.18 \mathrm{E}+00^{*}$ & $2.81 \mathrm{E}-02$ & $2.84 \mathrm{E}+00^{*}$ \\
\hline \multicolumn{7}{|c|}{ Lactantes de 3-6 meses } \\
\hline Cadmio & $6.84 \mathrm{E}-03$ & $2.11 \mathrm{E}-01$ & $1.59 \mathrm{E}-03$ & $3.41 \mathrm{E}-02$ & 4.00E-05 & 4.00E.05 \\
\hline Mercurio & $1.89 \mathrm{E}-01$ & $2.28 \mathrm{E}+00^{*}$ & $1.25 \mathrm{E}-01$ & $3.19 \mathrm{E}+00 *$ & 7.24E-02 & $4.44 \mathrm{E}+00^{*}$ \\
\hline Plomo & 7.33E-03 & $5.51 \mathrm{E}-02$ & $1.58 \mathrm{E}-02$ & $1.19 \mathrm{E}-01$ & $6.00 \mathrm{E}-04$ & $6.00 \mathrm{E}-04$ \\
\hline Suma & $2.03 \mathrm{E}-01$ & $2.55 \mathrm{E}+00^{*}$ & $1.42 \mathrm{E}-01$ & $3.34 \mathrm{E}+00^{*}$ & 7.24E-02 & $4.44 \mathrm{E}+00^{*}$ \\
\hline \multicolumn{7}{|c|}{ Lactantes de 6-12 meses } \\
\hline Cadmio & 8.97E-03 & 5.99E-01 & $2.12 \mathrm{E}-03$ & 9.87E-02 & $6.00 \mathrm{E}-05$ & $6.00 \mathrm{E}-05$ \\
\hline Mercurio & 2.32E-01 & $6.62 \mathrm{E}+00^{*}$ & $1.49 \mathrm{E}-01$ & $8.83 \mathrm{E}+00 *$ & $9.15 \mathrm{E}-02$ & $1.29 \mathrm{E}+01 *$ \\
\hline Plomo & $8.71 \mathrm{E}-03$ & $1.61 \mathrm{E}-01$ & $1.88 \mathrm{E}-02$ & $3.48 \mathrm{E}-01$ & $1.00 \mathrm{E}-03$ & $1.00 \mathrm{E}-03$ \\
\hline Suma & $2.50 \mathrm{E}-01$ & $7.38 \mathrm{E}+00^{*}$ & $1.70 \mathrm{E}-01$ & $9.28 \mathrm{E}+00^{*}$ & $9.15 \mathrm{E}-02$ & $1.29 \mathrm{E}+01 *$ \\
\hline \multicolumn{7}{|c|}{ Lactantes de $12-24$ meses } \\
\hline Cadmio & $2.93 \mathrm{E}-02$ & $3.42 \mathrm{E}-01$ & $6.74 \mathrm{E}-03$ & $5.52 \mathrm{E}-02$ & $8.00 \mathrm{E}-05$ & $8.00 \mathrm{E}-05$ \\
\hline Mercurio & 8.22E-01 & $3.76 \mathrm{E}+00^{*}$ & $5.60 \mathrm{E}-01$ & $5.14 \mathrm{E}+00 *$ & $3.27 \mathrm{E}-01$ & $7.31 \mathrm{E}+00^{*}$ \\
\hline Plomo & $3.15 \mathrm{E}-02$ & 8.98E-02 & $6.80 \mathrm{E}-02$ & $1.94 \mathrm{E}-01$ & $1.34 \mathrm{E}-03$ & $1.34 \mathrm{E}-03$ \\
\hline Suma & 8.83E-01 & $4.19 \mathrm{E}+00^{*}$ & $6.35 \mathrm{E}-01$ & $5.39 \mathrm{E}+00 *$ & $3.27 \mathrm{E}-01$ & $7.31 \mathrm{E}+00^{*}$ \\
\hline
\end{tabular}

*Valores de riesgo para el escenario menos favorable por encima del valor recomendado para el riesgo de efectos tóxicos no carcinogénicos

Los niveles mas altos de mercurio y plomo en las muestras de leche analizadas están por encima de $1.0 \mathrm{y}$ $2.0 \mu \mathrm{g} / \mathrm{kg}$, que son los límites máximos recomendados por la OMS para estos metales (Hermoza y Lomparte 2006). Los niveles de plomo y mercurio superan la norma de la OMS, hasta 21 y 20 veces, respectivamente. Para el cadmio, la Comisión del Codex Alimentarius de la Unión Europea estableció en la revisión de mayo de 2014 un contenido máximo en preparados lácteos para niños lactantes de $5 \mu \mathrm{g} / \mathrm{kg}$, por lo que las muestras 14 y 18 tomadas en Jarillón de Floralia en la comuna 6 de Cali, cumplen con el límite máximo establecido en esta norma europea. Las muestras del corregimiento El Hormiguero, que contienen las mayores concentraciones de cadmio, sobrepasan este valor hasta en un 523 $\%$. Estos resultados constituyen la primera evidencia del contenido de cadmio, mercurio y plomo en leche materna de madres residentes en la zona de estudio. La concentración de metales pesados en las muestras restantes está por debajo del límite de cuantificación y cumple con los límites máximos permitidos por la norma de la OMS y la Comisión del Codex Alimentarius.

\section{Evaluación de riesgo en salud de lactantes}

Para el riesgo no cancerígeno, la evaluación probabilística muestra que los valores asociados con el contenido de al menos uno de los metales pesados en leche materna, pudieran superar en cada lugar de muestreo y para cada subgrupo de lactantes los límites máximos permitidos por la USEPA $($ REN $\geq 1)$, tomando en cuenta el peor escenario o el mayor riesgo posible. En el caso del mejor escenario probabilístico, ninguno de los metales en ninguno de los lugares representaría un riesgo para la salud de los lactantes. En el caso del peor escenario, un valor de $R E N \geq 1$ indicaría que en la zona, los lactantes pudieran tener riesgo de padecer enfermedades como alteraciones en el desarrollo normal del cerebro, del sistema nervioso central, reducciones del cociente de inteligencia y malformaciones congénitas. En este caso, de acuerdo con la USEPA sería necesario tomar acciones en la zona para mitigar el riesgo potencial asociado con el consumo de leche materna con contenido de metales pesados. 
El mercurio fue el metal que más aportó al valor del riesgo no cancerígeno. Los valores aportados estuvieron entre el $89 \%$ y el $100 \%$ del valor total del riesgo en cada punto de muestreo y cada subgrupo de lactantes. Esto puede ser un indicador de los efectos en la salud que está generando la actividad minera, principalmente la extracción de oro, en zonas cercanas a los ríos tributarios del Cauca. En Colombia, diversos estudios han correlacionado la minería del oro con los aumentos en la concentración de mercurio en las fuente hídricas y en los organismos acuáticos, incluidos los peces (Díaz 2014). Estudios previos realizados en esta zona de estudio mostraron un alto contenido de cadmio, mercurio y plomo en dos especies de peces del río Cauca (Prochilodus magdalenae [bocachico] y Chaetostoma spp. [Corroncho]), las cuales son regularmente consumidas por las mujeres residentes en esta zona de estudio. Por lo tanto, las medidas correctivas deben incluir desde la descontaminación de las fuentes hídricas hasta una reducción en el consumo de pescado de este origen a cantidades que no representen un riesgo para la salud. Una aproximación sistémica al problema debería partir de entender las complejas interacciones de los ecosistemas con la sociedad y la salud. La lactancia materna es el mejor alimento para los primeros años de vida y los efectos potencialmente negativos asociados a los metales no deberían llevar a la conclusión de que sustituirla sea una medida adecuada. Lo que se necesita son políticas integrales que tengan en cuenta los contextos de los territorios y disminuyan las inequidades ambientales y de salud en estas comunidades vulnerables.

\section{AGRADECIMIENTOS}

Los autores agradecen a los habitantes de cada una de las zonas evaluadas por su disposición en la resolución de las encuestas poblacionales y la recolección de las muestras de leche materna. Asimismo, a la Universidad del Valle y el Fondo del Sistema General de Regalías del departamento del Valle del Cauca por la financiación de este proyecto.

\section{REFERENCIAS}

Álvarez S., Alan S., Jiménez F., Granados C. y Palacio J. (2012). Mercury concentrations in muscle and liver tissue of fish from marshes along the Magdalena river, Colombia. B. Environ. Contam. Tox. 89 (4), 836-840. DOI: $10.1007 / \mathrm{s} 00128-012-0782-9$
Artunduaga Y.P. (2014). Evaluación de riesgo en madres lactantes y determinación de $\mathrm{Hg}, \mathrm{Pb}$ y $\mathrm{Cd}$ en peces del río Cauca en la zona entre el corregimiento El Hormiguero en el municipio de Cali y la vereda El Paso de la Torre en el municipio de Yumbo. Tesis de Licenciatura. Departamento de Química, Facultad de Ciencias Naturales, Universidad del Valle. Cali, Colombia, 93 pp.

Castilla E., Mastroiacovo P., López-Camelo J., Saldarriaga W., Isaza C. y Orioli L. (2008). Sirenomelia and cyclopia cluster in Cali, Colombia. Am. J. Med. Genet. 146 (20), 2626-2636. DOI: 10.1002/ajmg.a.32506

Castro M. y Méndez M. (2008). Heavy metals: Implications associated to fish consumption. Environ. Toxicol. Phar. 26 (3), 263-271.

DOI: 10.1016/j.etap.2008.06.001

De Burbure C., Jean-Pierre B., Ariane L., Nisse C., Haguenoer M., Mutti A. y Smerhovský Z. (2006). Renal and neurologic effects of cadmium, lead, mercury, and arsenic in children: Evidence of early effects and multiple interactions at environmental exposure levels. Environ. Health. Persp. 114 (4), 584-590.

DOI: $10.1289 /$ ehp. 8202

Díaz F. (2014). Mercurio en la minería del oro: impacto en las fuentes hídricas destinadas para consumo humano. Rev. Salud Pub. 16 (6), 947-957.

DOI: 10.15446/rsap.v16n6.45406

Dietrich K., Omer B., Succop P., Hammond P. y Bornschein R. (1993). The developmental consequences of low to moderate prenatal and postnatal lead exposure: Intellectual attainment in the Cincinnati lead study cohort following school entry. Neurotoxicol. Teratol. 15 (1), 37-44. DOI: 10.1016/0892-0362(93)90043-N

Echeverry G., Zapata A., Paéz M., Méndez F. y Peña M. (2015). Valoración del riesgo en salud para un grupo poblacional de la ciudad de Cali-Colombia por exposición a $\mathrm{Pb}, \mathrm{Cd}, \mathrm{Hg}, 2-4 \mathrm{D}$ y Diuron debido al consumo de agua potable y alimentos. Biomédica 35 , 110-119. DOI: 10.7705/biomedica.v35i0.2464

FAO-OMS (2010). Informe de la consulta mixta de expertos sobre los riesgos y los beneficios del consumo de pescado. Organización de las Naciones Unidas para la Alimentación y la Agricultura-Organización Mundial de la Salud. Informe. Roma, Italia, 53 pp.

Figueroa R., Caicedo D., Echeverry G., Peña M. y Méndez F. (2017). Condición socioeconómica, patrones de alimentación y exposición a metales pesados en mujeres en edad fértil de Cali, Colombia. Biomédica 37 (3), 24-32. DOI: 10.7705/biomedica.v37i3.3286

Filigrana P., Gómez O. y Méndez F. (2011). Impacto de un sitio de disposición final de residuos sólidos en la salud respiratoria de los adultos mayores. Biomédica 31, 322-334. DOI: 10.7705/biomedica.v31i3.346 
García E., Pérez B., Fernández M., Pérez M., Gil E., Concha de P. e Iriso A. (2011). Mercury, lead and cadmium in human milk in relation to diet, lifestyle habits and sociodemographic variables in Madrid (Spain). Chemosphere 85 (2), 268-276.

DOI: 10.1016/j.chemosphere.2011.05.029

Gómez R., Filigrana P. y Méndez F. (2008). Descripción de la calidad del aire en el área de influencia del botadero de Navarro, Cali, Colombia. Colombia Médica 39 (3), 245-252.

Gracia H., Marrugo R. y Erasmo M. (2010). Contaminación por mercurio en humanos y peces en el municipio de Ayapel, Córdoba, Colombia. Revista Facultad Nacional Salud Pública 28,118-124.

DOI: 10.17533/udea.rfnsp

Grandjean P., Jørgensen J. y Weihe P. (1994). Human milk as a source of methylmercury exposure in infants. Environ. Health. Persp. 102 (1), 74-77.

DOI: $10.1289 /$ ehp. 9410274

Heidelore F., Cheble M., Pavesi T., Manfred T., Torres A., Castro S. y Santana V. (2010). Procedimiento operativo estándar para el muestreo de COP en leche materna. Manual. Montevideo, Uruguay, $20 \mathrm{pp}$.

Hermoza J. y Lomparte C. (2006). Determinación toxicológica de plomo en leche de madres lactantes del centro de salud San Juan Bosco de la Provincia Constitucional del Callao. Tesis de Licenciatura. Facultad de Farmacia y Bioquímica, Universidad Nacional Mayor de San Marcos. Lima, Perú, 40 pp.

LaKind J., Wilkins A. y Cheston M. (2004). Environmental chemicals in human milk: A review of levels, infant exposures and health, and guidance for future research. Toxicol. Appl. Pharm. 198 (2), 184-208.

DOI: $10.1016 /$ j.taap.2003.08.021

Mata L., Sánchez L. y Calvo M. (2003). Mercurio en leche. Revista de Toxicología, Asociación Española de Toxicología 20 (3), 176-181.

Mancera N. y Álvarez R. (2006). Current state of knowledge of the concentration of mercury and other heavy metals in fresh water fish in Colombia. Acta Biológica Colombiana 11 (1), 3-23.

Nazer H., Ariza P. e Isaza C. (2011). Defectos congénitos mayores en un hospital de tercer nivel en Cali. Rev. Salud. Pub. 13 (1), 152-162.

Olivero J. (2015). Efectos de la minería en Colombia sobre la salud humana. Minería en Colombia. Departamento Nacional de Planeación (DNP). Informe. Bogotá D.C, Colombia, $36 \mathrm{pp}$.

OPS (2010). Determinantes ambientales y sociales de la salud. Organización Panamericana de la Salud. McGraw-Hill Interamericana Editores, Washington, D.C., EUA, 545 pp.
OMS (2010). La alimentación del lactante y del niño pequeño. Capítulo modelo para libros de texto dirigidos a estudiantes de medicina y otras ciencias de la salud. Organización Mundial de la Salud, Washington, D.C., EUA, $87 \mathrm{pp}$.

Orioli M., Mastroiacovo P., López J., Saldarriaga W., Isaza C., Aiello H., Zarante I. y Castilla E. (2009). Clusters of sirenomelia in South America. Clinical and Molecular Teratology 85 (2), 112-118. DOI: 10.1002/bdra.20492

Páez M., Varona M., Díaz S. y Castro R. (2011). Evaluación del riesgo en humanos por plaguicidas en tomate cultivado con sistemas tradicional y BPA (Buenas Prácticas Agrícolas). Revista Facultad de Ciencias 15,153-167.

Restrepo L., Rodríguez H. y Valencia D. (2016). Caracterización del consumo de pescado y mariscos en población universitaria de la ciudad de MedellínColombia. Universidad y Salud 18 (2), 257-265.

Solomon G. y Weiss M. (2002). Chemical contaminants in breast milk: Time trends and regional variability. Environ. Health. Persp. 110 (6), 339-347.

DOI: $10.1289 /$ ehp.021100339

Thompson J. y Bannigan J. (2008). Cadmium: Toxic effects on the reproductive system and the embryo. Reprod. Toxicol. 25 (3), 304-315.

DOI: $10.1016 /$ j.reprotox.2008.02.001

Tobergte D. y Curtis S. (2013). Microwave assisted acid digestion of sileceous and organically based matrices. United States Environmental Protection Agency, Washington, D.C., EUA, 10 pp.

USEPA (1998). Method 7010: graphite furnace atomic absorption spectrophotometry. United States Environmental Protection Agency, Washington, D.C., EUA, 20 pp.

USEPA (2001). Risk assessment guidance for superfund volume I human health risk evaluation. United States Environmental Protection Agency, Washington, D.C., EUA, 93 pp.

USEPA (2005). Guidelines for carcinogen risk assessment. United States Environmental Protection Agency, Washington, D.C., EUA, 30 pp.

Vieira C., Morais S., Ramos S., Delerue D. y Oliveira M. (2011). Mercury, cadmium, lead and arsenic levels in three pelagic fish species from the Atlantic ocean: Intra and inter specific variability and human health risks for consumption. Food. Chem. Toxicol. 49 (4), 923-932. DOI: $10.1016 /$ j.fct.2010.12.016

Vivas H., Arboleda A., Sánchez R., Benítez N., Bravo E., Soto A., Jiménez A., Muñoz A. y Larmat F. (2014). Mutagenicity evaluation caused by heavy metals found in Cauca river water in the city of Cali, Colombia. Revista Colombiana de Química 43 (2), 18-24. DOI: 10.15446/rev.colomb.quim.v43n2.53119 